\title{
IKERLANAK
}

\section{PROFICIENCY, ATTITUDE AND CONVENTIONS IN MINORITY LANGUAGES}

by

Annick Laruelle, Noemía Navarro and Ramón Escobedo

2015

Working Paper Series: IL. 92/15

Departamento de Fundamentos del Análisis Económico I

Ekonomi Analisiaren Oinarriak I Saila

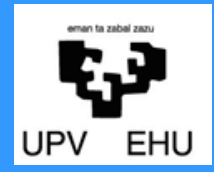

University of the Basque Country 


\title{
Proficiency, Attitude and Conventions in Minority Languages*
}

\author{
Annick Laruelleł Noemí Navarro ${ }^{\S}$ and Ramón Escobedo * $^{\ddagger}$
}

\begin{abstract}
In this paper we study a simple mathematical model of a bilingual community in which all agents are fluent in the majority language but only a fraction of the population has some degree of proficiency in the minority language. We investigate how different distributions of proficiency, combined with the speakers' attitudes towards or against the minority language, may influence its use in pair conversations.
\end{abstract}

${ }^{*}$ The paper was inspired by a working group at the department of Economics I of the University of the Basque Country. The authors benefitted from these discussion. The help of Sergio Faria is gratefully acknowledged. The first author acknowledges the support of the Spanish Ministerio de Economía y Competitividad under project ECO2012-31346, and of the Departamento de Educación, Política Lingüística y Cultura from the Basque Government (Research Group IT568-13). The second author acknowledges financial support from IKERBASQUE, Basque Foundation for Science, through a Research Fellowship, and is grateful to Fundamentos de Análisis Económico I department at UPV/EHU for their hospitality and great research atmosphere. The third author acknowledges the support of the Advanced Grant NUMERIWAVES/FP7-246775 of the European Research Council Executive Agency, the BERC 2014-2017 program of the Basque Government, the FA9550-15-1-0027 of AFOSR, the MTM2014-52347 and MTM2011-29306 Grants and the Severo Ochoa program SEV-2013-0323 of the MINECO, and a Humboldt Award at the University of Erlangen-Nürnberg.

${ }^{\dagger}$ BRiDGE, Foundation of Economic Analysis I, University of the Basque Country (UPV/EHU) Avenida Lehendakari Aguirre, 83, E-48015 Bilbao, Spain, annick.laruelle@ehu.es.

${ }^{\ddagger}$ IKERBASQUE, Basque Foundation of Science, 48011, Bilbao, Spain.

${ }^{\S}$ Groupe de Recherche en Économie Théorique et Appliquée (GREThA), Université de Bordeaux. Avenue Léon Duguit 33608 Pessac cedex, France, noemi.navarro@u-bordeaux.fr.

ॠBCAM: Basque Center for Applied Mathematics, Alameda de Mazarredo 14, 48009 Bilbao, Spain.

"BRiDGE, Applied Economics IV, University of the Basque Country (UPV/EHU) Avenida Lehendakari Aguirre, 83, E-48015 Bilbao, Spain.

**AEPA-Euskadi, Puente de Deusto 7, 48014 Bilbao, Spain. 


\section{Introduction}

The European Union has a long tradition of linguistic diversity within its Member States. According to the 2006 Eurobarometer only nine ${ }^{1}$ out of the twenty-eight States can be considered as linguistically homogeneous, i.e. with at least $97 \%$ of the population reporting a common mother tongue. Twelve states ${ }^{2}$ report figures of between $90 \%$ and $96 \%$, and a further $\operatorname{six}^{3}$ between $70 \%$ and $89 \%$. In Belgium $56 \%$ of the population report Dutch as their mother tongue and 38\%report French. With the exceptions of Belgium, Estonia and Luxembourg there is always a majority language spoken by the whole population.

The European Charter for Regional or Minority Languages, which entered into force in 1998, seeks to assure respect for, protection and promotion of regional and minority languages in Europe. It defines regional or minority languages as languages that are "traditionally used within a given territory of a State by nationals of that State who form a group numerically smaller than the rest of the State's population; and different from the official language(s) of that State". A minority language is not necessarily a language at risk of extinction though many of them are classified as either vulnerable or endangered according to the UNESCO Atlas of the World Languages in Danger.

The use of regional or minority languages varies greatly from one language and one territory to another. In Catalonia (Spain) more than $60 \%$ of the population are able to speak and write in Catalan, and 36\% report that they use Catalan exclusively on a daily basis, while another further $12 \%$ use both Catalan and Spanish on a daily basis (Generalitat de Catalunya, 2009). In the Basque Autonomous Community (Spain), the reported knowledge of Basque is around 35\%, while the use is around 13\% (Basque Government, 2011). In Ireland the percentage of the population who can speak Irish is $40.6 \%$ and $2 \%$ speak Irish on a daily basis outside the education system (Government of Ireland, 2012). The relationship between knowledge and use of languages is thus far from linear.

Abrams and Strogatz (2003) propose a macroscopic model of language competition which predicts that two languages cannot coexist stably - one will eventually drive the other to extinction. These authors arrive at the conclusion that increasing language status is crucial in order to prevent the decline of minority languages. Their model however assumes that individuals are monolingual. Minett and Wang (2008) incorporate bilingualism and language transmission but their model yields the same extinction conclusion.

The objective of this paper is to study language competition at a microscopic level: we seek to understand the mechanism that determines which language is chosen for conversation when various languages coexist. One fundamental distinction between languages in competition is that the majority language is spoken by all, while minority languages are spoken by only a fraction of the population. The model is kept as simple as possible: we concentrate on bilingual conversations and start with a static model (that is, we disregard from learning concerns). Each speaker is characterised by two features considered by Fishman (1991, p.49) as being of overriding importance: attitude and competence (or proficiency). We follow van Parijs (2007) and consider that communication exchanges yield value for speakers through these two different dimensions: efficiency and expressive value. The proficiency of the speakers participating in the conversation affects the efficiency in exchanging information. The attitude or motivation of the speakers participating in the conversation gives value for expressive reasons. We take the view that proficiency and attitude in generating worth or value are equally important. Note that the efficiency of a conversation is determined by the speaker with the lower proficiency (the "maximin law of communication" van Parijs, 2007). By

\footnotetext{
${ }^{1}$ Croatia, Cyprus, Czech Republic, Denmark, Greece, Hungary, Malta, Poland, Portugal.

${ }^{2}$ Austria (96\%), Bulgaria (90\%), Finland (94\%), France (93\%), Germany (90\%), Ireland (94\%), Italy (95\%), Netherlands (96\%), Romania (95\%), Slovenia (95\%), Sweden (95\%) and the United Kingdom (92\%).

${ }^{3}$ Estonia (82\%), Latvia (73\%), Lithuania (88\%), Luxembourg (77\%), Slovakia (88\%), Spain (89\%).
} 
contrast the expressive value of a conversation depends exclusively on the speaker's attitude towards the language. Once the value of a conversation is fixed for each speaker, a social convention selects the language to be spoken. That social convention is accepted by the whole of society as a social norm, and therefore has some reasonable properties. We focus on conventions that are anonymous, unanimous and monotone. ${ }^{4}$

The main question is whether the mechanism above described is neutral in the sense introduced by May (1952). In our context the mechanism is neutral if it does not favour either language. Individual preferences are neutral: if the names of the languages are changed the preferred language is not changed. In a conversation the proficiency of the partner also matters when determining the value of a conversation because the value of a conversation depends on the proficiency of both speakers. This introduces a bias in favour of the majority language: preferences in conversations are no longer neutral. The is due exlusively to the fact that the value of the information exchange suffers from a (negative) externality due to a lack of proficiency in the minority language. It is shown that no convention can offset this bias in favour of the majority language, i.e. even the convention that is most favourable to the minority language cannot offset the bias in favour of the majority language that is generated by the interaction of the proficiency of speakers in a conversation.

The rest of the paper is organised as follows. In Section 2 we study the individual preferences. Section 3 shows the bias that arises in conversations. Section 4 reviews the convention, and Section 5 shows that there is a bias in the choice of language for any convention. Section 6 concludes with some discussion. The Appendix contains the proofs.

\section{Individual preference for a language}

Consider a population of speakers that can make use of two linguistically distant languages $A$ and $B$ in (for the sake of simplicity bilateral) communication exchanges. The majority language $A$ is spoken by the whole population, while not all individuals are able to maintain a conversation in the minority language $B$. In this section we describe the relative (positive or negative) preference for language $B$ with respect to language $A$.

Speakers positively value a communication exchange in a given language for two reasons: information exchange and expressive reasons. The quantity and quality of information exchanged is critically determined by the proficiency of the speaker: the higher the proficiency the more information is exchanged and the better its quality is. ${ }^{5}$ Languages are also used as carriers of cultural identity and of expressive meanings beyond the pure informative statements. ${ }^{6}$ The importance of the language as an expressive meaning is captured by the attitude of the speakers towards the language. Hence, the speaker's preference for one language over the other results from the interaction of these two parameters, proficiency and attitude.

For the sake of simplicity we assume that all individuals have the same level of proficiency in $A$ and the same attitude towards $A$, and choose language $A$ as a benchmark. A speaker can then be characterised by $\vec{x}=(m, p)$ where $m$ denotes the individual's relative attitude towards $B$, and $p$ denotes her or his relative proficiency in $B$. We assume $-1 \leq p \leq 1$ and $-1<m<1$ with the following interpretation: monolingual individuals (who only speak $A$ ) have $p=-1$, bilingual individuals have $-1<p \leq 1$. An individual with $-1 \leq p<0$ is more proficient in $A$, and an individual with $0<p \leq 1$ is more proficient in $B$. An individual equally proficient in both languages has $p=0$. Similarly the interpretation of $m=0$ is that the attitude towards both languages is identical. The quantity $m$ is strictly positive $(0<m<1)$ if the

\footnotetext{
${ }^{4} \mathrm{~A}$ convention is anonymous if the identities of the individuals do not matter. A convention is unanimous if whenever both speakers have the same preferred language, the convention chooses it. Finally, a convention is monotone if more intensity in the speakers' preference for a language does not hinder its use.

${ }^{5}$ In a conversation information exchange also depends on the proficiency of the interlocutor.

${ }^{6}$ Philippe van Parijs (2007) calls this feature of language use "expressive reasons" or "expressive concerns".
} 
individual has a more positive attitude towards $B$, while $-1<m<0$ reflects a more positive attitude towards $A$. Any speaker can be identified by her/his characteristics $\vec{x}=(m, p)$. The space of speakers is referred to as $\mathcal{X}=]-1,1[\times[-1,1]$.

Both characteristics have positive effects on the preference for the minority language compared to the majority language: the more proficient or the more positive the attitude the more preferred the minority language is relative to the majority language. We consider that the effects of proficiency and attitude on language preference are separable additively, hence allowing for cases where proficiency and attitude do not go hand in hand: a monolingual individual may have a positive attitude towards the use of the minority language or an individual with higher proficiency in the minority language may have a negative attitude towards the minority language. We take the view that proficiency and attitude are at least partially substitutes: the preference for a language can be maintained if one characteristic increases and the other decreases. For instance a more positive attitude towards the minority language can offset a lower level of proficiency. For the sake of simplicity, we consider that the two characteristics are perfect substitutes. The individual utility of the minority language relatively to the majority language (or individual utility for short) is the sum of the relative attitude and relative proficiency. Denoting by $u_{i i}$ the individual utility of speaker $\vec{x}_{i}=\left(m_{i}, p_{i}\right)$ the following is obtained

$$
u_{i i}=m_{i}+p_{i} .
$$

This is interpreted as $\vec{x}_{i}$ 's relative preference for language $B$ over language $A$. If $u_{i i}<0$ speaker $\vec{x}_{i}$ prefers language $A$, she/he is indifferent if $u_{i i}=0$, and prefers language $B$ if $u_{i i}>0$. The absolute value of $u_{i i}$ measures the intensity of the (positive or negative) preference. Note that a monolingual individual (i.e. if $\left.p_{i}=-1\right)$ always prefers $A\left(u_{i i}<0\right.$ given that $\left.m_{i}<1\right)$.

Neutrality requires that both languages be treated equally, i.e. if languages were labelled differently the results would remain identical. If we had chosen $B$ as the benchmark instead of $A$, the relative attitude and proficiency levels of individuals would just have the opposite sign: a speaker would be characterised by $-\vec{x}_{i}=\left(-m_{i},-p_{i}\right)$ instead of $\vec{x}_{i}=\left(m_{i}, p_{i}\right)$. The space of characteristics is neutral given that $\vec{x}_{i} \in \mathcal{X} \Longleftrightarrow-\vec{x}_{i} \in \mathcal{X}$. One implication of neutrality is that in a uniformly distributed population (that is, same density of individuals in $\mathcal{X}$ ), both languages are preferred by the same number of speakers.

Now consider the neutrality of preferences. If $B$ were taken as reference, the preference for the majority language would be reflected by a positive utility, and negative utility would mean a preference for the minority language. Preferences are neutral if the following equivalence holds: the utility of any speaker $\vec{x}_{i}$ is strictly positive if and only if that of speakers $-\vec{x}_{i}$ is strictly negative. For any speaker $\vec{x}_{i}$ let $\bar{u}_{i i}$ denote the utility of $-\vec{x}_{i}$. Given that $-\vec{x}_{i}=$ $\left(-m_{i},-p_{i}\right)$, it emerges that $\bar{u}_{i i}=-m_{i}-p_{i}$ and neutrality is trivially satisfied: for any $\vec{x}_{i} \in \mathcal{X}$ it holds that $u_{i i}>0 \Longleftrightarrow \bar{u}_{i i}<0$.

\section{Biased preferences in a conversation}

The high proficiency of a speaker is worth nothing in a conversation if the other speaker is monolingual or has a poor knowledge of the language. The exchange of information is limited by the speaker with the lower proficiency. Formally, in a conversation between speakers $\vec{x}_{i}=$ $\left(m_{i}, p_{i}\right)$ and $\vec{x}_{j}=\left(m_{j}, p_{j}\right)$ the exchange is constrained in terms of proficiency by $\min \left\{p_{i}, p_{j}\right\}$. By contrast the expressive value of the conversation is intrinsic to the individual and is not affected by the characteristics of the other participant. The relative preference of individual $\vec{x}_{i}$ for a language in conversation with $\vec{x}_{j}$ is determined by attitude of $\vec{x}_{i}\left(m_{i}\right)$ and the proficiency of the conversation $\left(\min \left\{p_{i}, p_{j}\right\}\right)$. In a conversation between $\vec{x}_{i}$ and $\vec{x}_{j} u_{i j}$ denotes speaker $\vec{x}_{i}$ 's utility. This gives the following

$$
u_{i j} \stackrel{\text { def }}{=} m_{i}+\min \left\{p_{i}, p_{j}\right\}
$$


If $u_{i j}<0$ speaker $\vec{x}_{i}$ prefers language $A$ in a conversation with $\vec{x}_{j}$, she/he is indifferent if $u_{i j}=0$, and she/he prefers language $B$ if $u_{i j}>0$. The absolute value of $u_{i j}$ measures the intensity of the (positive or negative) preference.

The utilities obtained by speakers $\vec{x}_{i}$ and $\vec{x}_{j}$ in a conversation generally differ: $u_{i j} \neq u_{j i}$ when $m_{i} \neq m_{j}$. If $u_{i j} \neq u_{j i}$ both speakers share the same preference for a language if $u_{i j} u_{j i}>0$ or one speaker prefers the minority language while the other prefers the majority language if $u_{i j} u_{j i}<0$. Similarly speaker $\vec{x}_{i}$ may have different preferences for languages in conversation with different partners $\vec{x}_{j}$ and $\vec{x}_{k}$ : this may give $u_{i j} u_{i k}<0$.

For any conversation between $\vec{x}_{i}$ and $\vec{x}_{i}$ the values of $u_{i j}$ and $u_{j i}$ vary in the interval ] $-2,2$ [. All individuals are assumed to have the same utility function $u$ :

$$
\left.u: \mathcal{X}^{2} \rightarrow\right]-2,2\left[, \quad\left(\vec{x}_{i}, \vec{x}_{j}\right) \rightarrow u\left(\vec{x}_{i}, \vec{x}_{j}\right)=u_{i j} .\right.
$$

The value $u_{i i}$ is the utility obtained by $\vec{x}_{i}$ in a conversation with another speaker with identical characteristics $\left(m_{i}, p_{i}\right)$. It is also the individual utility of $\vec{x}_{i}$ as defined in (1).

Obvious properties can be derived from (1) and (2).

1. The highest utility of $\vec{x}_{i}$ 's is reached when conversing with a partner with identical characteristics: $u_{i j} \leq u_{i i}$ for any $\vec{x}_{j} \in \mathcal{X}$, or

$$
u_{i i}=\max _{\vec{x}_{j} \in \mathcal{X}}\left\{u_{i j}\right\}
$$

2. If $\vec{x}_{i}$ individually prefers $A$ (that is, if $u_{i i}<0$ ), then this preference is kept in any conversation: $u_{i j}<0$ for any $\vec{x}_{j} \in \mathcal{X}$.

3. Speaker $\vec{x}_{i}$ prefers language $A$ in a conversation with $\vec{x}_{j}$ if either $\vec{x}_{i}$ or $\vec{x}_{j}$ does not have sufficient proficiency: $u_{i j}<0$ if $p_{j}<-m_{i}$ or $p_{i}<-m_{i}$.

4. Speaker $\vec{x}_{i}$ individually prefers $B$ but prefers $A$ in a conversation with $\vec{x}_{j}$ if $\vec{x}_{j}$ has a lower proficiency than $\vec{x}_{i}\left(p_{j}<p_{i}\right)$ and the attitude of $\vec{x}_{i}$ towards the minority language is not sufficiently large to offset this lower proficiency: $p_{j}<-m_{i}<p_{i}$.

5. Speaker $\vec{x}_{i}$ prefers language $B$ in a conversation with $\vec{x}_{j}$ only if both $\vec{x}_{i}$ and $\vec{x}_{j}$ have sufficient proficiency: $u_{i j}>0$ if $p_{j}>-m_{i}$ and $p_{i}>-m_{i}$.

6. If $\vec{x}_{i}$ prefers $B$ in a conversation with any $\vec{x}_{j}$, then $\vec{x}_{i}$ individually prefers $B: u_{i j}>0 \Rightarrow$ $u_{i i}>0$.

Thus, two conditions must be satisfied for a speaker to prefer the minority language in a conversation: her/his attitude has to offset her/his own lack of proficiency and that of the other participant; by contrast, failure to meet just one condition is enough for the speaker to prefer the majority language. Preferring the majority language in a conversation with a speaker of the same level is a sufficient condition to prefer the majority language in any conversation, while preferring the minority language in a conversation with a speaker of the same level is not a sufficient condition to prefer the minority language in any conversation.

As a result although the preferences of individuals are neutral, neutrality is lost for preferences in conversations. Neutrality would require the signs of the utilities in a conversation between $-\vec{x}_{i}$ and $-\vec{x}_{j}$ to be the opposites of the signs of the utilities in the conversation between $\vec{x}_{i}$ and $\vec{x}_{j}$. Using the following notation

$$
\bar{u}_{i j} \stackrel{\text { notation }}{=} u\left(-\vec{x}_{i},-\vec{x}_{j}\right) \text {, }
$$

the preferences of $\vec{x}_{i}$ 's would be neutral if $u_{i j} \bar{u}_{i j}<0$ or $u_{i j}=\bar{u}_{i j}=0$ for any $\vec{x}_{j}$. It is shown below that preferences are not neutral as there are some $\left(\vec{x}_{i}, \vec{x}_{j}\right) \in \mathcal{X}^{2}$ such that $u_{i j} \bar{u}_{i j}>0$. 
Nevertheless whenever $u_{i j}>0$ it holds that $\bar{u}_{i j}<0$. This means that there is a bias in favour of the majority language: whenever $\vec{x}_{i}$ prefers $B$ in a conversation with $\vec{x}_{j}$ it results that $-\vec{x}_{i}$ prefers $A$ in a conversation with $-\vec{x}_{j}$. By contrast it may be that $\vec{x}_{i}$ prefers $A$ in a conversation with $\vec{x}_{j}$ and $-\vec{x}_{i}$ also prefers $A$ in a conversation with $-\vec{x}_{j}$.

Definition 1 Preferences in conversations are

1. A-biased if $u_{i j}>0 \Rightarrow \bar{u}_{i j}<0$ for any $\left(\vec{x}_{i}, \vec{x}_{j}\right) \in \mathcal{X}^{2}$,

2. B-biased if $u_{i j}<0 \Rightarrow \bar{u}_{i j}>0$ for any $\left(\vec{x}_{i}, \vec{x}_{j}\right) \in \mathcal{X}^{2}$.

Preferences in conversations are neutral if they are both $A$-biased and $B$-biased. The bias is systematic if preferences are biased toward one language and not towards the other. The following proposition sums up what is stated above (proof in the Appendix).

Proposition 2 Preferences in conversations are systematically A-biased.

Neutrality holds for individual preferences but ceases to hold for preferences in conversation: there is a systematic bias in favour of the majority language. The implication is that although both languages are individually preferred in a uniformly distributed population by the same number of speakers (that is, there are as many speakers with $u_{i i}<0$ as $u_{i i}>0$ ) more individuals prefer the majority language in conversations. Indeed the speakers who individually prefer the majority language keep their preferences in all conversations, while those who individually weakly prefer the minority language switch their preference if the interlocutor lacks sufficient proficiency. The scale of the bias depends on how speakers are distributed within $\mathcal{X}$. In particular it depends on the speaker's individual preference and the interlocutor's proficiency, as shown in the following proposition (proof of which is given in the Appendix).

Proposition 3 Given a speaker $\vec{x}_{i} \in \mathcal{X}$,

1. If $u_{i i}<0$, then $u_{i j}<0$ for any $\vec{x}_{j} \in \mathcal{X}$.

2. If $u_{i i}=0$, then $u_{i j} \leq 0$ for any $\vec{x}_{j} \in \mathcal{X}$. The equality $u_{i j}=0$ holds if $p_{j} \geq-m_{i}$.

3. If $u_{i i}>0$, then (a) $u_{i j}<0$ for any $\vec{x}_{j}$ such $p_{j}<-m_{i}$, (b) $u_{i j}>0$ for any $\vec{x}_{j}$ such $p_{j}>-m_{i}(c) u_{i j}=0$ if $p_{j}=-m_{i}$.

\section{Linguistic Conventions}

So far we have explored the speakers' preferences for one language or the other. In a conversation between $\vec{x}_{i}$ and $\vec{x}_{j}$ the respective preferences of the speakers are given by $u_{i j}$ and $u_{j i}$. The utilities space is denoted by $\mathcal{U}=]-2,2[\times]-2,2\left[\right.$. An element of the space $\left(u_{i j}, u_{j i}\right) \in \mathcal{U}$ represents a pair of utilities obtained in a conversation. A linguistic convention $\mathcal{C}$ associates either language $A$ or $B$ with any pair $\left(u_{i j}, u_{j i}\right)$.

$$
\mathcal{C}: \mathcal{U} \rightarrow\{A, B\},\left(u_{i j}, u_{j i}\right) \rightarrow \mathcal{C}\left(u_{i j}, u_{j i}\right)=A \text { or } B
$$

We thus assume that there is a linguistic convention which is accepted by the whole society as a social norm. In all conversations the same rule is applied to select the language. This rule is exclusively based on the respective utilities of both speakers in the conversation. A convention satisfies certain reasonable properties. In order to be accepted as a convention a rule should satisfy (at least) three properties: 
1. Anonymity: $\forall\left(u_{i j}, u_{j i}\right) \in \mathcal{U}: \mathcal{C}\left(u_{i j}, u_{j i}\right)=\mathcal{C}\left(u_{j i}, u_{i j}\right)$.

2. Unanimity: $\forall\left(u_{i j}, u_{j i}\right) \in \mathcal{U}$ such that $\left(u_{i j}, u_{j i}\right) \neq(0,0)$ :

$$
\begin{aligned}
& \text { if } u_{i j} \geq 0 \text { and } u_{j i} \geq 0 \Rightarrow \mathcal{C}\left(u_{i j}, u_{j i}\right)=B, \\
& \text { if } u_{i j} \leq 0 \text { and } u_{j i} \leq 0 \Rightarrow \mathcal{C}\left(u_{i j}, u_{j i}\right)=A .
\end{aligned}
$$

3. Monotonicity: $\forall\left(u_{i j}, u_{j i}\right),\left(v_{i j}, v_{j i}\right) \in \mathcal{U}$ :

$$
\begin{array}{ll}
\text { if } \mathcal{C}\left(u_{i j}, u_{j i}\right)=A, \text { then: } & u_{i j} \geq v_{i j} \text { and } u_{j i} \geq v_{j i} \Rightarrow \mathcal{C}\left(v_{i j}, v_{j i}\right)=A, \\
\text { if } \mathcal{C}\left(u_{i j}, u_{j i}\right)=B, \text { then: } & u_{i j} \leq v_{i j} \text { and } u_{j i} \leq v_{j i} \Rightarrow \mathcal{C}\left(v_{i j}, v_{j i}\right)=B .
\end{array}
$$

Anonymity means that the identities of the speakers do not matter. This is a reasonable property as long as individuals have no role in the conversation. ${ }^{7}$ Unanimity requires that if both speakers share the same preference for a language then the convention selects the preferred language, and if one speaker is indifferent and the other strictly prefers one language, then the convention selects the language preferred by the speaker with strict preference. In our context this is equivalent to the Pareto efficiency condition: if the choice of the minority (majority) makes at least one speaker better off and does not make the other speaker worse off then the minority (majority) language is chosen. Finally, monotonicity states that if a convention selects one language for two given speakers then it must to select the same language for two other speakers with the same or stronger preferences for that language.

A convention $\mathcal{C}$ can be graphically represented by dividing the $\mathcal{U}$-space into the subspaces $\mathcal{U}_{A}(\mathcal{C})$ and $\mathcal{U}_{B}(\mathcal{C})$ where $A$ and $B$ are chosen respectively. Anonymity requires $\mathcal{U}_{A}(\mathcal{C})$ and $\mathcal{U}_{B}(\mathcal{C})$ to be symmetric with respect to the first diagonal. Unanimity requires the first quadrant of $\mathcal{U}$ to be included in $\mathcal{U}_{B}(\mathcal{C})$ and the third quadrant $\mathcal{U}$ to be included in $\mathcal{U}_{A}(\mathcal{C})$. Monotonicity requires $\mathcal{U}_{A}(\mathcal{C})$ and $\mathcal{U}_{B}(\mathcal{C})$ to be connected. Graphic examples of conventions are shown in Figure 1.

Figure 1 about here

Obviously the larger $\mathcal{U}_{B}(\mathcal{C})\left(\mathcal{U}_{A}(\mathcal{C})\right)$ is the more favourable the convention is towards the minority (majority) language. Convention $\mathcal{C}_{1}$ is more favourable to $B$ than convention $\mathcal{C}_{2}$ (or convention $\mathcal{C}_{2}$ is more favourable to $A$ than convention $\left.\mathcal{C}_{1}\right)$ if $\mathcal{U}_{B}\left(\mathcal{C}_{2}\right) \subset \mathcal{U}_{B}\left(\mathcal{C}_{1}\right)$.

The convention that is most favourable to $B$ is denoted by $\mathcal{C}_{B}$. It selects the minority language unless both speakers share the same preference for the majority language:

$$
\mathcal{C}_{B}\left(u_{i j}, u_{j i}\right)=\left\{\begin{array}{l}
A \text { if } u_{i j} \leq 0, u_{j i} \leq 0 \text { and }\left(u_{i j}, u_{j i}\right) \neq(0,0) \\
B \text { otherwise. }
\end{array}\right.
$$

Similarly $\mathcal{C}_{A}$ can be defined as the convention that is most favourable to the majority language.

$$
\mathcal{C}_{A}\left(u_{i j}, u_{j i}\right)=\left\{\begin{array}{l}
B \text { if } u_{i j} \geq 0, u_{j i} \geq 0 \text { and }\left(u_{i j}, u_{j i}\right) \neq(0,0) \\
A \text { otherwise }
\end{array}\right.
$$

We now focus on the condition of neutrality and the space of utilities when the minority language is taken as the benchmark instead of the majority one. If $\left(u_{i j}, u_{j i}\right)$ is the pair of utilities when $\mathrm{A}$ is taken as benchmark, then the pair of utilities if B were taken as benchmark would be given by $\left(-u_{i j},-u_{j i}\right)$. The space of utilities is neutral given that $\left(u_{i j}, u_{j i}\right) \in \mathcal{U} \Longleftrightarrow\left(-u_{i j},-u_{j i}\right) \in \mathcal{U}$. A convention is neutral if the choice of language for $\left(-u_{i j},-u_{j i}\right)$ is the opposite of the choice for $\left(u_{i j}, u_{j i}\right)$. That is, if, for all $\left(u_{i j}, u_{j i}\right) \in \mathcal{U}$,

$$
\mathcal{C}\left(u_{i j}, u_{j i}\right)=A \Longleftrightarrow \mathcal{C}\left(-u_{i j},-u_{j i}\right)=B
$$

\footnotetext{
${ }^{7}$ For instance if one individual is a citizen and the other an institution, identity may matter. Citizens may have the right to be attended by an institution in their preferred language.
} 
This condition reduces to $\mathcal{C}\left(u_{i j},-u_{i j}\right) \neq \mathcal{C}\left(-u_{i j}, u_{i j}\right)$ whenever $u_{i j}+u_{j i}=0$. This is incompatible with anonymity, which requires $\mathcal{C}\left(u_{i j}, u_{j i}\right)=\mathcal{C}\left(u_{j i}, u_{i j}\right)$. In order to avoid this incompatibility, we only require equivalence for $\left(u_{i j}, u_{j i}\right) \in \mathcal{U}$ such that $u_{i j}+u_{j i} \neq 0$.

If only one part of the equivalence (6) holds for all $\left(u_{i j}, u_{j i}\right) \in \mathcal{U}$ the convention is systematically biased towards one language or the other.

Definition $4 A$ convention $\mathcal{C}$ is $A$-biased if for any pair $\left(u_{i j}, u_{j i}\right) \in \mathcal{U}$ such that $u_{i j}+u_{j i} \neq 0$ the following holds true:

$$
\mathcal{C}\left(u_{i j}, u_{j i}\right)=B \Rightarrow \mathcal{C}\left(-u_{i j},-u_{j i}\right)=A .
$$

$A$ convention $\mathcal{C}$ is B-biased if the following holds true:

$$
\mathcal{C}\left(u_{i j}, u_{j i}\right)=A \Rightarrow \mathcal{C}\left(-u_{i j},-u_{j i}\right)=B .
$$

It is clear that $\mathcal{C}_{A}$ is systematically $A$-biased, while $\mathcal{C}_{B}$ is systematically $B$-biased. Other examples are given in Figure 1, along with an example of a neutral convention and an example of a non biased convention, i.e. a convention such that there are $\left(u_{i j}, u_{j i}\right),\left(v_{i j}, v_{j i}\right) \in \mathcal{U}$ with $\mathcal{C}\left(u_{i j}, u_{j i}\right)=\mathcal{C}\left(-u_{i j},-u_{j i}\right)=A$ and $\mathcal{C}\left(v_{i j}, v_{j i}\right)=\mathcal{C}\left(-v_{i j},-v_{j i}\right)=B$.

A neutral convention can be characterised up to pairs $\left(u_{i j}, u_{j i}\right)$ such that $u_{i j}+u_{j i}=0$, as shown by the following proposition (see the Appendix for proof).

Proposition 5 A convention $\mathcal{C}$ is neutral if and only if it meets the following conditions:

$$
\begin{aligned}
& \text { (i) } u_{i j}+u_{j i}<0 \Rightarrow \mathcal{C}\left(u_{i j}, u_{j i}\right)=A, \\
& \text { (ii) } u_{i j}+u_{j i}>0 \Rightarrow \mathcal{C}\left(u_{i j}, u_{j i}\right)=B .
\end{aligned}
$$

Interestingly, a neutral convention can be seen as a utilitarian convention because it chooses the language that maximises the sum of utilities. Furthermore, a neutral convention also satisfies the Rawlsian criterion because it selects the language in such a way that it benefits the speaker with the highest degree of preference (the one who would be worse off if her/his less preferred language were chosen).

\section{Biased choice of Language}

Given a pair of speakers $\left(\vec{x}_{i}, \vec{x}_{j}\right)$, the preferences in the conversation are determined by $u_{i j}=u\left(\vec{x}_{i}, \vec{x}_{j}\right)$ and $u_{j i}=u\left(\vec{x}_{j}, \vec{x}_{i}\right)$ as defined in (3). The language is chosen by applying a convention to $\left(u_{i j}, u_{j i}\right)$. The choice of language under a convention can thus be seen as a mechanism that associates the choice of a language with any pair of characteristics $\left(\vec{x}_{i}, \vec{x}_{j}\right) \in \mathcal{X}^{2}$.

The question of neutrality in the choice of the language can be addressed. The $\mathcal{X}^{2}$ space is obviously neutral. The relevant question is whether the pair $\left(-\vec{x}_{i},-\vec{x}_{j}\right)$ will always result in a different choice of language than the pair $\left(\vec{x}_{i}, \vec{x}_{j}\right)$. The answer depends on the convention. It seems clear that the convention that is the most favourable to the majority language will result in a choice in favour of the majority language: indeed the preferences in conversation are $A$-biased. Can the convention in favour of the minority language offset the bias in preferences and result in a neutral choice of language?

To answer that question a formal definition of neutral choice of language is needed. That definition is the natural extension of the definitions above: the language choice is neutral under a convention if it is $A$-biased and $B$-biased. Biases are defined using notation (5). 
Definition 6 The choice of a language under convention $\mathcal{C}$ is A-biased if for any pair $\left(\vec{x}_{i}, \vec{x}_{j}\right)$ such that $u_{i j}+u_{j i} \neq 0$ the following holds:

$$
\mathcal{C}\left(u_{i j}, u_{j i}\right)=B \Rightarrow \mathcal{C}\left(\bar{u}_{i j}, \bar{u}_{j i}\right)=A .
$$

The choice of a language is B-biased under convention $\mathcal{C}$ if for any pair $\left(\vec{x}_{i}, \vec{x}_{j}\right)$ such that $u_{i j}+u_{j i} \neq 0$ the following holds:

$$
\mathcal{C}\left(u_{i j}, u_{j i}\right)=A \Rightarrow \mathcal{C}\left(\bar{u}_{i j}, \bar{u}_{j i}\right)=B .
$$

As shown in the following proposition the answer to the question of whether a neutral choice of language can be obtained is no.

Proposition 7 Whatever the convention $\mathcal{C}$, the choice of language under $\mathcal{C}$ is never B-biased.

The proof in the Appendix shows that the choice of language is never $B$-biased as there are $\left(\vec{x}_{i}, \vec{x}_{j}\right)$ with $\mathcal{C}\left(u_{i j}, u_{j i}\right)=\mathcal{C}\left(\bar{u}_{i j}, \bar{u}_{j i}\right)=A$. Note that a stronger property could be proved: for any $\vec{x}_{i} \in \mathcal{X}$, there is $\vec{x}_{j} \in \mathcal{X}$ with $\mathcal{C}\left(u_{i j}, u_{j i}\right)=\mathcal{C}\left(\bar{u}_{i j}, \bar{u}_{j i}\right)=A$. That is, whatever the convention and whoever the speaker $\vec{x}_{i}$, there is $\vec{x}_{j}$ such that the choice of language is the majority language in their conversation and in the conversation between $-\vec{x}_{i}$ and $-\vec{x}_{j}$.

Preferences in conversations are $A$-biased, even if individual preferences are neutral. This bias cannot be offset by the convention, not even by the convention that is most favourable to the minority language, $\mathcal{C}_{B}$. The choice of language under $\mathcal{C}_{B}$ is not $B$-biased, and thus not neutral. By contrast the choice of language under $\mathcal{C}_{A}$ is systematically $A$-biased. The following proposition (proof of which is in the Appendix) shows a stronger result: other conventions result in an $A$-biased choice of language.

Proposition 8 The choice of language under any A-biased convention is A-biased.

One direct consequence of this proposition is that the choice of language under any neutral convention is systematically $A$-biased.

In a uniformly distributed population, both languages are individually preferred by the same number of speakers but more individuals prefer the majority language in conversations. If conversations between pairs of individuals are random all conventions select the majority language more often. The frequency depends on the distribution of the population within $\mathcal{X}$, and on the convention. We have this final proposition.

Proposition 9 For speakers $\vec{x}_{i} \neq \vec{x}_{j} \in \mathcal{X}$ such that $\left(u_{i j}, u_{j i}\right) \neq(0,0)$ the following is found: for any convention $\mathcal{C}$

1. if $\max \left\{-m_{i},-m_{j}\right\} \leq \min \left\{p_{i}, p_{j}\right\}$ then $\mathcal{C}\left(u_{i j}, u_{j i}\right)=B$,

2. if $\min \left\{-m_{i},-m_{j}\right\}>\min \left\{p_{i}, p_{j}\right\}$ then $\mathcal{C}\left(u_{i j}, u_{j i}\right)=A$.

The proof is omitted as it is a direct consequence of Lemma 10 (see Appendix). Note that $\min \left\{-m_{i},-m_{j}\right\} \leq \min \left\{p_{i}, p_{j}\right\} \leq \max \left\{-m_{i},-m_{j}\right\}$ then $u_{i j} . u_{j i} \leq 0$, and the choice of the language depends on the convention.

\section{Discussion}

Our model shows that a minority language in contact with a majority language also suffers from a disadvantage from a static point of view. This disadvantage comes from the externality originated by the interlocutor's lack of proficiency as compared to one's own attitude for the 
minority language. The speaker's preference in a conversation crucially depends on her/his attitude to the minority language. The larger a speaker's attitude is, the lower the proficiency that she/he may accept from a partner in a conversation. By contrast a speaker with an individual preference for the majority language always prefers the majority language in any conversation.

The importance of attitude in minority language is well-known among linguists. As put by Grenoble (2013, p. 797): "Clearly more positive attitudes toward the language tend to strengthen its usage; and more negative attitudes to weaken it". Our research provides an understanding the mechanism that yields this effect. It also shows that a positive attitude is more important for those who have high proficiency levels (that is, those who individually prefer the minority language) than for those who have low proficiency levels. In particular it shows that the phenomenon depends on how the population is located in the spectrum of proficiency and attitude. Our research also suggests that the key element is preferences in conversations. Once these are formed and present a bias no convention can offset this bias, not even the convention that selects the minority language unless both individuals prefer the majority language.

Further work includes an application of this model to case studies. Collecting data is not without difficulties as "there is usually no practical alternative to either collecting self report data about them via "scales' or questionnaires" (see Fishman 1991, p. 49). Our model does not preclude the possibility of introducing dynamics. For example, an individual may decide to learn the language or not, and if the language is not used competence may decrease. We guess that the long-term effect of these dynamic elements will compound the (static) bias in favour of the majority language that we have identified here, in each period of the dynamic setting. Nevertheless, a dynamic model might help suggest possible avenues for policy intervention. For example, as already suggested, a government could try to promote the language in order to increase motivation.

\section{Appendix}

The Appendix is devoted to proofs.

\section{Proposition 2}

Proof. For any $\left(\vec{x}_{i}, \vec{x}_{j}\right) \in \mathcal{X}^{2}$ we compute $u_{i j}$ and $\bar{u}_{i j}$ for all possible values of $m_{i}, p_{i}, m_{j}, p_{j}$.

(i) If $p_{i}>p_{j}$ then $u_{i j}=m_{i}+p_{j}$ and $\bar{u}_{i j}=-m_{i}-p_{i}$. (a) If $-m_{i}<p_{j}$ then $u_{i j}>0$ and $\bar{u}_{i j}<0$, (b) if $p_{j} \leq-m_{i} \leq p_{i}$ then $u_{i j} \leq 0$ and $\bar{u}_{i j}<0$ and (c) If $-m_{i} \geq p_{i}$ then $u_{i j}<0$ and $\bar{u}_{i j} \geq 0$.

(ii) If $p_{i}=p_{j}$ then $u_{i j}=m_{i}+p_{i}$ and $\bar{u}_{i j}=-m_{i}-p_{i}$. (a) If $-m_{i}<p_{i}$ then $u_{i j}>0$ and $\bar{u}_{i j}<0$, (b) if $-m_{i} \geq p_{i}$ then $u_{i j} \leq 0$ and $\bar{u}_{i j} \geq 0$.

(iii) If $p_{i}<p_{j}$ then $u_{i j}=m_{i}+p_{i}$ and $\bar{u}_{i j}=-m_{i}-p_{j}$. (a) If $-m_{i}<p_{i}$ then $u_{i j}>0$ and $\bar{u}_{i j}<0$, (b) if $p_{i} \leq-m_{i} \leq p_{j}$ then $u_{i j} \leq 0$ and $\bar{u}_{i j}<0$ and (c) If $-m_{i} \geq p_{j}$ then $u_{i j}<0$ and $\bar{u}_{i j}>0$.

It can be seen that if $u_{i j}>0$ then $\bar{u}_{i j}<0$ while we may have $u_{i j}<0$ and $\bar{u}_{i j}<0$.

\section{Proposition 3}

Proof. (i) If $u_{i i}<0$ then $u_{i j}<0$ for any $\vec{x}_{j} \in \mathcal{X}$ given that (4) holds. (ii) If $u_{i i}=0$ again $u_{i j} \leq 0$ for any $\vec{x}_{j} \in \mathcal{X}$. Now, if $p_{j}<-m_{i}$, then $u_{i j}<0$, while if $p_{j} \geq-m_{i}, u_{i j}=0$. (iii) If $u_{i i}>0$, then $p_{i}+m_{i}>0$ so the condition for $u_{i j}>0$ is that $m_{i}+p_{j}>0$. Then, if $m_{i}+p_{j}=0$, $u_{i j}=0$, and if $m_{i}+p_{j}<0, u_{i j}<0$.

\section{Proposition 5}

Proof. It is easy to check that a function $\mathcal{C}$ satisfying (9) is anonymous, unanimous and monotone, and furthermore, it verifies (7) and (8) (and hence neutral). The second step 
consist of proving that if a convention $C$ satisfies (7) and (8), on top of the properties of anonymity, unanimity and monotonicity, then it satisfies (9). By contradiction, suppose $\left.\left(u_{i j}, u_{j i}\right) \in\right]-2,2[\times]-2,2\left[\right.$ is such that $\left(u_{i j}, u_{j i}\right) \neq 0, u_{i j}+u_{j i}>0$ but there is a convention $C$ satisfying (7) and (8) together with anonymity, unanimity, and monotonicity with $\mathcal{C}\left(u_{i j}, u_{j i}\right)=A$. Given that $\mathcal{C}$ satisfies unanimity, it has to be that $u_{i j} u_{j i}<0$. Fix $u_{i j}>0$ and $u_{j i}<0$. By anonymity of $\mathcal{C}, \mathcal{C}\left(u_{j i}, u_{i j}\right)=\mathcal{C}\left(u_{i j}, u_{j i}\right)=A$. Since $\mathcal{C}$ satisfies (8), $\mathcal{C}\left(-u_{j i},-u_{i j}\right)=B$ because $\mathcal{C}\left(u_{j i}, u_{i j}\right)=A$. But $u_{i j}+u_{j i}>0$ implies $u_{i j}>-u_{j i}$ and $u_{j i}>-u_{i j}$, and so $\left(-u_{j i},-u_{i j}\right)<\left(u_{i j}, u_{j i}\right)$. By monotonicity of $\mathcal{C}$, if $\mathcal{C}\left(u_{i j}, u_{j i}\right)=A$ then $\mathcal{C}\left(-u_{j i},-u_{i j}\right)=A$, a contradiction with $\mathcal{C}\left(-u_{j i},-u_{i j}\right)=B$. Similarly (by contradiction) it can be shown that if $\mathcal{C}$ is anonymous, unanimous and monotone, and furthermore, it verifies (7) and (8), then it has to choose $A$ whenever $u_{i j}+u_{j i}<0$.

The proofs of the Propositions 7, 8 and $\mathbf{9}$ are based on the following lemma, that determines the choice of language for any pair $\vec{x}_{i} \neq \vec{x}_{j} \in \mathcal{X}$ such that $\left(u_{i j}, u_{j i}\right) \neq(0,0)$.

Lemma 10 For speakers $\vec{x}_{i} \neq \vec{x}_{j} \in \mathcal{X}$ such that $\left(u_{i j}, u_{j i}\right) \neq(0,0)$ the following properties hold:

1. If $\max \left\{-m_{i},-m_{j}\right\} \leq \min \left\{p_{i}, p_{j}\right\}$ then $\mathcal{C}\left(u_{i j}, u_{j i}\right)=B$ and $\mathcal{C}\left(\bar{u}_{i j}, \bar{u}_{j i}\right)=A$.

2. If $\min \left\{-m_{i},-m_{j}\right\} \leq \min \left\{p_{i}, p_{j}\right\} \leq \max \left\{-m_{i},-m_{j}\right\} \leq \max \left\{p_{i}, p_{j}\right\}$ then $u_{i j} . u_{j i} \leq 0$ and $\mathcal{C}\left(\bar{u}_{i j}, \bar{u}_{j i}\right)=A$.

3. If $\min \left\{p_{i}, p_{j}\right\} \leq \min \left\{-m_{i},-m_{j}\right\} \leq \max \left\{-m_{i},-m_{j}\right\} \leq \max \left\{p_{i}, p_{j}\right\}$ then $\mathcal{C}\left(u_{i j}, u_{j i}\right)=$ $\mathcal{C}\left(\bar{u}_{i j}, \bar{u}_{j i}\right)=A$.

4. If $\min \left\{p_{i}, p_{j}\right\} \leq \min \left\{-m_{i},-m_{j}\right\} \leq \max \left\{p_{i}, p_{j}\right\} \leq \max \left\{-m_{i},-m_{j}\right\}$ then and $\mathcal{C}\left(u_{i j}, u_{j i}\right)=$ $A$ and $\bar{u}_{i j} \cdot \bar{u}_{j i} \leq 0$.

5. If $\min \left\{-m_{i},-m_{j}\right\} \geq \max \left\{p_{i}, p_{j}\right\}$ then $\mathcal{C}\left(u_{i j}, u_{j i}\right)=A$ and $\mathcal{C}\left(\bar{u}_{i j}, \bar{u}_{j i}\right)=B$.

6. If $\min \left\{-m_{i},-m_{j}\right\} \leq \min \left\{p_{i}, p_{j}\right\}<\max \left\{p_{i}, p_{j}\right\} \leq \max \left\{-m_{i},-m_{j}\right\}$ then $u_{i j} . u_{j i} \leq 0$ and $\bar{u}_{i j} \cdot \bar{u}_{j i} \leq 0$.

Proof. If $\max \left\{-m_{i},-m_{j}\right\} \leq \min \left\{p_{i}, p_{j}\right\}$ then it holds $u_{i j}=m_{i}+\min \left\{p_{i}, p_{j}\right\} \geq 0, u_{j i}=$ $m_{j}+\min \left\{p_{i}, p_{j}\right\} \geq 0$. Then by unanimity $\mathcal{C}\left(u_{i j}, u_{j i}\right)=B$. The following is also obtained: $\bar{u}_{i j}=-m_{i}+\min \left\{-p_{i},-p_{j}\right\} \leq 0, u_{j i}=-m_{j}+\min \left\{-p_{i},-p_{j}\right\} \leq 0$, and by unanimity $\mathcal{C}\left(\bar{u}_{i j}, \bar{u}_{j i}\right)=A$. The other properties are proved similarly.

\section{Proposition 7}

Proof. Choose $\vec{x}_{i}=\left(m_{i}, p_{i}\right)$ and $\vec{x}_{j}=\left(m_{j}, p_{j}\right)$ such that $-1 \leq p_{i}<-m_{j} \leq-m_{i}<p_{j} \leq 1$. By Lemma 10 the following is obtained $\mathcal{C}\left(u_{i j}, u_{j i}\right)=A$ and $\mathcal{C}\left(\bar{u}_{i j}, \bar{u}_{j i}\right)=A$.

\section{Proposition 8}

Proof. All possible pairs $\left(\vec{x}_{i}, \vec{x}_{j}\right) \in \mathcal{X}^{2}$ such that $u_{i j}+u_{j i} \neq 0$ are considered. (i) If $\vec{x}_{i}=\vec{x}_{j}$ it is obtained $\mathcal{C}\left(u_{i j}, u_{j i}\right)=\mathcal{C}\left(u_{i i}, u_{i i}\right)$. For $u_{i i} \neq 0 \mathcal{C}\left(u_{i i}, u_{i i}\right)=B$ if $u_{i i}>0$. Then $\mathcal{C}\left(\bar{u}_{i i}, \bar{u}_{i i}\right)=A$. (ii) Lemma 10 guarantees that if $\mathcal{C}\left(u_{i j}, u_{j i}\right)=B$ then $\mathcal{C}\left(\bar{u}_{i j}, \bar{u}_{j i}\right)=A$ for all $\vec{x}_{i} \neq \vec{x}_{j} \in \mathcal{X}$ such that $\min \left\{-m_{i},-m_{j}\right\} \leq \min \left\{p_{i}, p_{j}\right\}<\max \left\{p_{i}, p_{j}\right\} \leq \max \left\{-m_{i},-m_{j}\right\}$ does not hold. (iii) If $-m_{i} \leq p_{i}<p_{j} \leq-m_{j}$ then $u_{i j}=m_{i}+p_{i} \geq 0, u_{j i}=m_{j}+p_{i}<0, \bar{u}_{i j}=-m_{i}-p_{j}<$ $0, \bar{u}_{j i}=-m_{j}-p_{j} \leq 0$. If the convention is $A$-biased, if $\mathcal{C}\left(m_{i}+p_{i}, m_{j}+p_{i}\right)=B$ then $\mathcal{C}\left(-m_{i}-p_{j},-m_{j}-p_{j}\right)=A$. (iv) The cases $-m_{j} \leq p_{i}<p_{j} \leq-m_{i},-m_{i} \leq p_{j}<p_{i} \leq-m_{j}$ and $-m_{j} \leq p_{j}<p_{i} \leq-m_{i}$ are proved in a similar way than (iii). 


\section{References}

[1] Abrams, D.M. and S.H. Strogatz, 2003, "Modeling the dynamics of language death." Nature 424: p.900.

[2] European Commission, 2006, Europeans and their Languages, Special Eurobarometer 243, TNS Opinion and Social.

[3] Fishman, J. A. , 1991, Reversing Language Shift: Theoretical and Empirical Foundations of Assistance to Threatened Languages, Clevedon, England. Multilingual matters.

[4] Generalitat de Catalunya, 2009, Enquesta d'usos lingüístics de la població 2008, Secretaria de Política Lingüística i l'Institut d'Estadística de Catalunya 1a. edició: Barcelona, juny del 2009, B-26.115-2009 versió digital.

[5] Gabinete de Prospección Sociológica, Eusko Jaurlaritza-Gobierno Vasco, 2011, Euskera: uso, actitudes, políticas, Department of education, political linguistics and culture of the Basque Government, Vitoria-Gasteiz.

[6] Government of Ireland, Central Statistics Office, 2012, Profile 9, What we know, Government Publications Sales Office, Sun Alliance House,Molesworth Street, Dublin 2, ISBN 978-1-4064-2671-7.

[7] Grenoble, L. A., 2013, Language revitalization. In Robert Bayley, Richard Cameron, \& Ceil Lucas, eds., The Oxford Handbook of Sociolinguistics, 792-811. Oxford: Oxford University Press.

[8] May, K. O, 1952, A set of independent necessary and sufficient conditions for simple majority decision, Econometrica 20(4), 680-684.

[9] van Parijs, P., 2007, Europe's Linguistic Challenge, in "The Language Question in Europe and Diverse Societies", Dario Castiglione and Chris Longman (eds.). Oxford: Hart, 2007, pp. 215-251. 


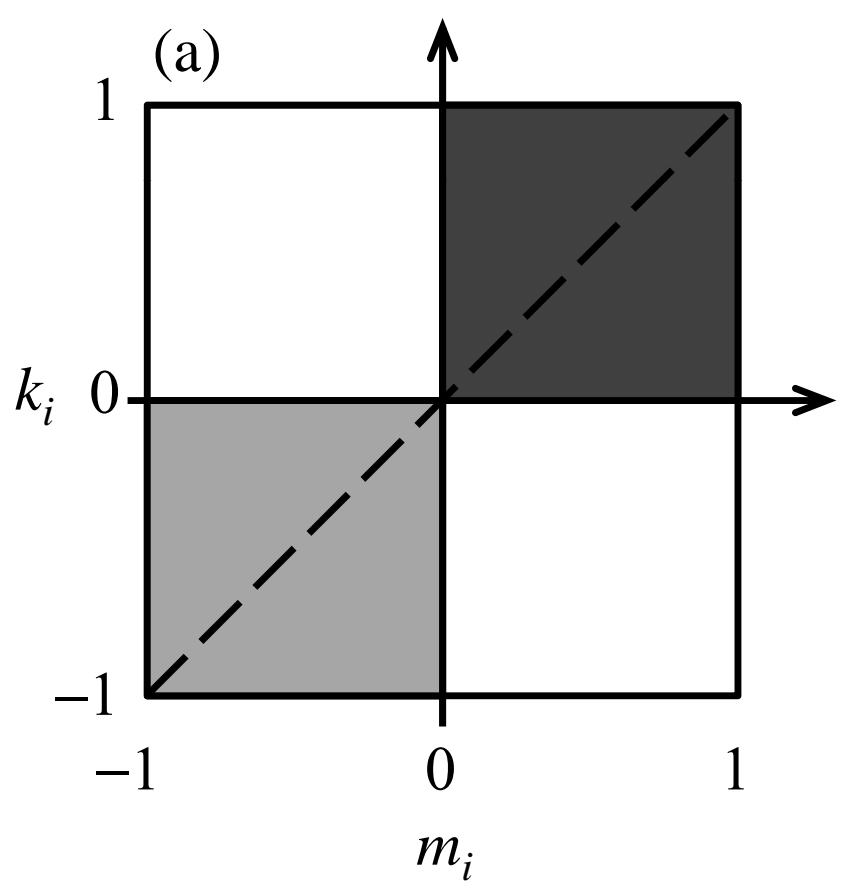

(d)

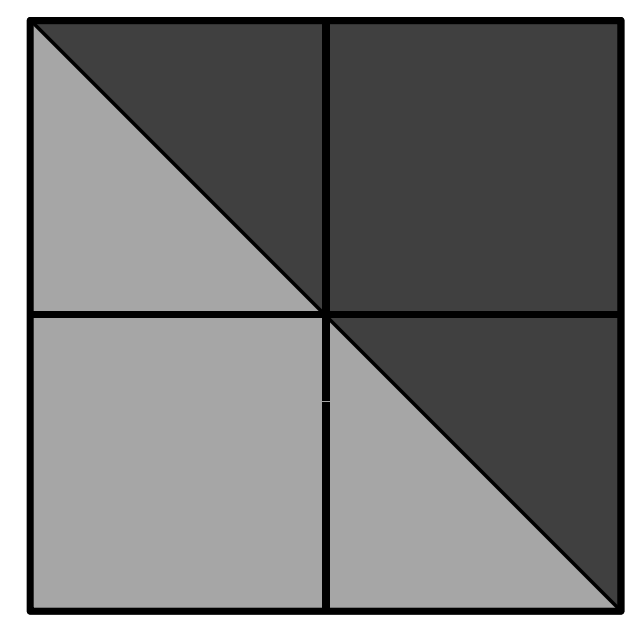

Neutral convention (b)

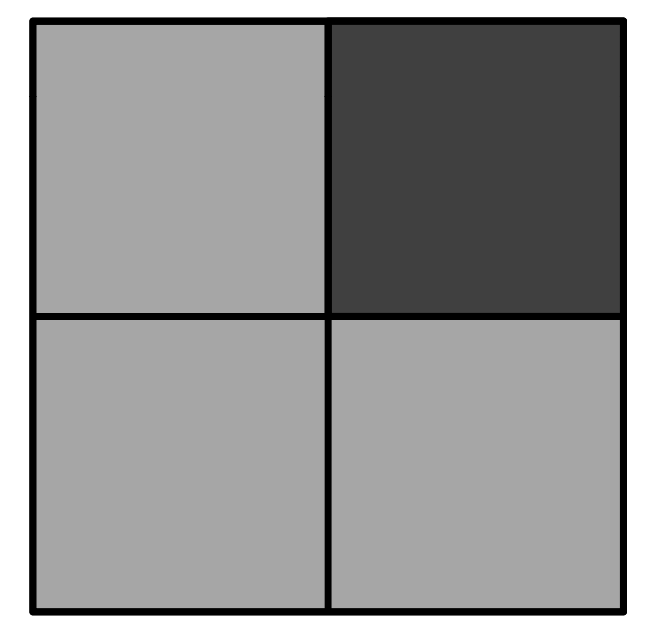

Convention $C_{\mathrm{A}}$

(e)

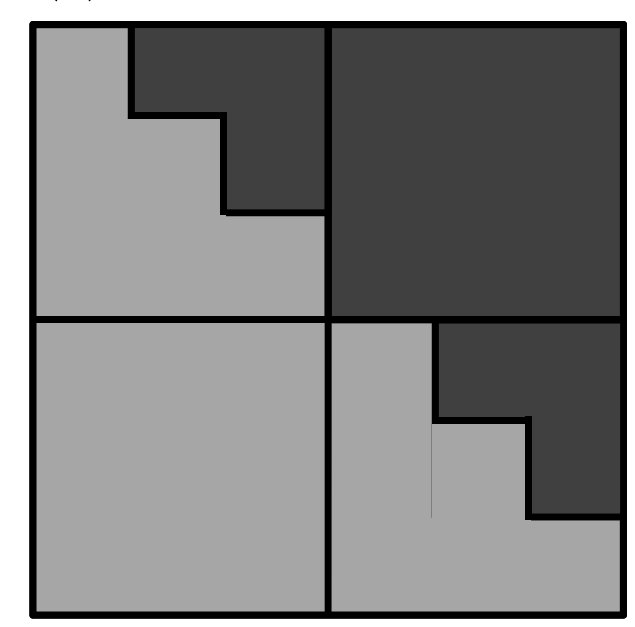

A-biased convention (c)

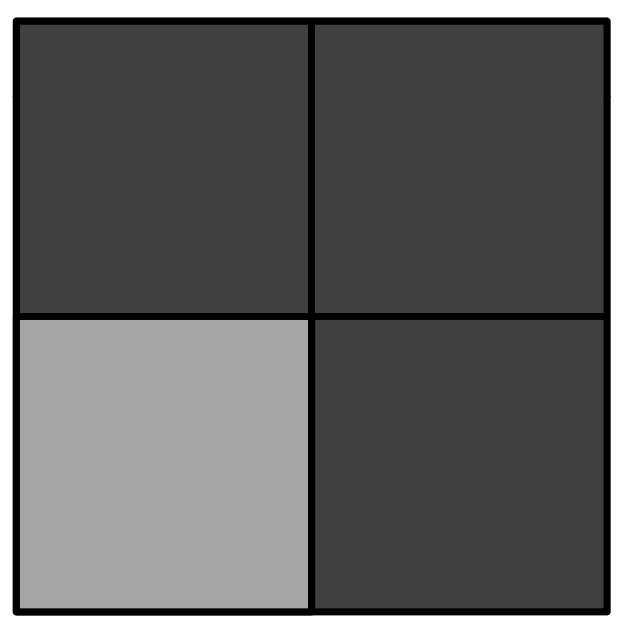

Convention $C_{\mathrm{B}}$

(f)

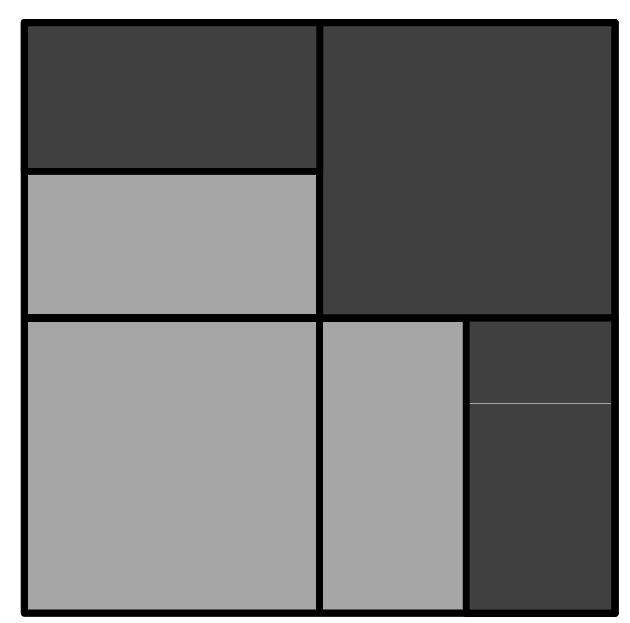

Non biased convention 